\title{
The Association Between Computer Use and Cognition Across Adulthood: Use It So You Won't Lose It?
}

\author{
Patricia A. Tun and Margie E. Lachman \\ Brandeis University
}

\begin{abstract}
Understanding the association between computer use and adult cognition has been limited until now by self-selected samples with restricted ranges of age and education. Here we studied effects of computer use in a large national sample $(N=2,671)$ of adults aged $32-84$, assessing cognition with the Brief Test of Adult Cognition by Telephone (Tun \& Lachman, 2005) and executive function with the Stop and Go Switch Task (Tun \& Lachman, 2008). Frequency of computer activity was associated with cognitive performance after controlling for age, sex, education, and health status: That is, individuals who used the computer frequently scored significantly higher than those who seldom used the computer. Greater computer use was also associated with better executive function on a task-switching test, even after controlling for basic cognitive ability as well as demographic variables. These findings suggest that frequent computer activity is associated with good cognitive function, particularly executive control, across adulthood into old age, especially for those with lower intellectual ability.
\end{abstract}

Keywords: cognition, computer use, cognitive activity, executive function, task switching

Although computers have become an integral part of daily life in the modern world, relatively little is known about the "digital divide" between computer users and nonusers and how this relates to mental performance across adulthood. Research shows that higher cognitive abilities predict success in computer training (Ownby, Czaja, Loewenstein, \& Rubert, 2008). Also, computer users tend to be younger, with higher levels of education and income (Fox, 2006). Studies based on convenience samples of volunteers report that computer users have higher cognitive status than nonusers (e.g., Czaja et al., 2006), but the nature of this association remains unresolved: It is not clear whether brighter people choose to use the computer, or using the computer might help maintain cognitive abilities. The consequences of this issue are significant, as researchers do not yet know whether continued engagement with computers will widen the gap in cognitive ability across socioeconomic gradients, and perhaps leave some individuals at risk of being marginalized in modern society, and/or whether computer use might actually help keep adults mentally sharp as they age. Here we explore the association between computer use and

This article was published Online First August 2, 2010.

Patricia A. Tun, Volen National Center for Complex Systems and Department of Psychology, Brandeis University; Margie E. Lachman, Department of Psychology, Brandeis University.

This research was supported by National Institute on Aging Grant PO1 AG20166 to conduct a longitudinal follow-up of the MIDUS (Midlife in the U.S.) investigation. The original study was supported by the John D. and Catherine T. MacArthur Foundation Research Network on Successful Midlife Development. We are grateful to the University of Wisconsin Survey Center and MIDUS II staff for outstanding work in data collection, and to Chandra Murphy for assistance with data scoring and analysis.

Correspondence concerning this article should be addressed to Patricia A. Tun, Volen Center MS013, Brandeis University, Waltham, MA 02454. E-mail: tun@brandeis.edu cognitive performance in a large national sample of adults ranging in age from 32 to 84 with a wide range of education and socioeconomic status.

Computer use is an interactive activity that presents special challenges to older adults (e.g., Charness \& Boot, 2009) ranging from increased difficulty in psychomotor ability in using the computer mouse to sensory and cognitive abilities such as speed of searching for icons, to issues with learning, memory, and executive function, all of which may be compromised with age (Charness \& Boot, 2009). Nevertheless, surveys show that adults of all ages have embraced the computer and the Internet, with a dramatic increase in use by adults over 65 in the last decade, from $15 \%$ in 2001 (Fox, 2001) to 32\% in 2006 (Fox, 2006). Older adults increasingly rely on the Internet for e-mail communication and information on health care (Kane, Boston, \& Chivers, 2007), products, travel, and online banking (Fox, 2006).

The current study makes a unique contribution as the first major national, population-based investigation of the association between computer activity and cognitive performance across adulthood. Although an important earlier study of adult volunteers (Czaja et al., 2006) reported significant age differences in computer use as a function of cognitive abilities as well as attitudes, the selective nature of that sample leaves unanswered questions about the general aging population, as do other studies using smaller convenience samples (e.g., Umemuro, 2004). Our research advances previous work by drawing from a probability sample of men and women ranging from young adulthood through midlife to old age. This study includes 2,671 individuals from the Midlife in the U.S. (MIDUS II) national sample of adults, with a wide range of geographic, ethnic, socioeconomic, and educational backgrounds. The MIDUS data include a selection of measures that allows researchers to investigate the association of computer use and cognition controlling for demographic, socioeconomic status, and health variables. 
We examined the hypothesis that greater use of the computer will be associated with higher levels of cognitive performance across adulthood, particularly in age-sensitive cognitive domains such as executive function. Support for this comes from a recent study (Basak, Boot, Voss, \& Kramer, 2008) that found that adults who were trained on complex video games showed an improvement in executive function tasks such as task switching, compared to a control group who did not train.

A fairly substantial research literature has supported the "use it or lose it" notion that remaining mentally active in old age may help preserve good cognitive function (Schooler, 2007), perhaps in the form of a cognitive reserve (Fritsch et al., 2007; Stern et al., 2005), or of increased complexity of neuronal synapses (Saczynski et al., 2008). A number of studies have demonstrated that participation in cognitively challenging activities is associated with a higher level of cognitive performance (Hultsch, Hammer, \& Small, 1993; Wilson et al., 1999), or a reduced rate of cognitive decline (Hultsch, Hertzog, Small, \& Dixon, 1999; Verghese et al., 2003; Wilson et al., 2002). This association may be especially important for individuals with low educational attainment (Lachman, Agrigoraei, Murphy, \& Tun, 2010).

However, others have argued that those who are better educated and more cognitively able simply choose to remain mentally active across adulthood (Salthouse, 2006). A recent intervention study of computer Internet training found few effects on cognitive performance in older adults, but suggested that those with higher cognitive ability may have used the computer more extensively (Slegers, van Boxtel, \& Jolles, 2009). Thus, an alternative outcome of this study would be that any increase in cognitive performance associated with computer use might be reduced after controlling for other factors such as education, income, and health status. In order to examine these hypotheses, we first examined the association between frequency of computer use and performance on the cognitive tasks from the Brief Test of Adult Cognition by Telephone (BTACT; Lachman \& Tun, 2008; Tun \& Lachman, 2005, 2006), which assesses key cognitive domains such as memory, processing speed, and reasoning. Next, we specifically focused on the association between computer activity and executive function, which plays a critical role in coordinating the multiple tasks involved in using a computer. We examined whether computer use is associated with better task-switching performance on the Stop and Go Switch Task (SGST; Tun \& Lachman, 2008), after controlling for basic cognitive ability as well as demographic and health variables. Thus, this analysis represents a conservative test of the hypothesis that more frequent computer use is associated with better executive function in adults who are similar in basic intellectual abilities as well as age, sex, education, and health status. If controlling for basic intellectual ability eliminates a positive association between computer use and task switching, this would suggest that higher functioning individuals are simply more likely to use the computer. In contrast, if a positive association with task-switching latencies remained, this would suggest that computer use is associated with better performance in specific cognitive domains.

\section{Method}

\section{Participants}

Participants were drawn from 4,175 adults who were part of the cognitive telephone interview in the second Midlife in the United
States (MIDUS II) survey of adults ages 32-84. Telephone interviews were conducted in 1995-1996 and 2005-2006, and the cognitive tests and computer questions were administered at Time 2. A national probability sample of households with at least one telephone was selected initially in 1995 using random digit dialing for the MIDUS survey (Brim, Ryff, \& Kessler, 2004). The sample of 7,108 noninstitutionalized adults was stratified to achieve equal sex distribution and an age distribution with the greatest number between 40 and 60 . Approximately 10 years later, $75 \%$ of the sample $(N=4,963)$, adjusted for mortality, participated in the second wave of the study; the mean Time 1 age of the longitudinal sample did not differ in age from those who dropped out, $(M=$ 46.21 vs. 46.84 years; $t[7039]=0.78)$, but the longitudinal sample had more education $(M=14.00$ vs. 13.09 years; $t[7085]=13.09$, $p<.001)$ and was more likely to be female, $\chi^{2}(1)=17.91, p<$ .001 .

After completing the larger survey at Time 2, which included the questions about demographics and computer use, 4,175 participants later carried out a cognitive battery by telephone. Of these, 52 were excluded from further analyses because they did not speak English regularly, and 320 because they reported neurological problems including stroke and Parkinson's disease. We omitted an additional 176 cases due to technical problems, 803 who did not have complete data for variables assessed here, and 153 who did not meet the accuracy criterion on the task-switching test (described in the following section).

The remaining sample of 2,671 participants had complete cognitive data and task-switching latencies. Their age ranged from 32 to 84 with a mean of 55.28 years $(S D=11.86)$, and $54.9 \%$ were women. All spoke English on a regular basis. The racial composition of this sample was 92.4\% Caucasian, 3.4\% African American, and $4.2 \%$ other races. The final sample of 2,671 participants who were selected was compared to those who were not selected from the Time 2 sample of 4,175. Those selected were younger than those who were not included ( 55.54 vs. 56.31 years of age at testing Time $2 ; t[4464]=2.05, p<.05)$, had more education (14.30 vs. 14.02 years; $t[4490[=3.06, p<.01)$, and had a lower percentage of women $\left(52.3 \%\right.$ vs. $56.5 \% ; \chi^{2}[1[=9.7, p<.01)$. The nonselected group had a racial composition of $86.3 \%$ Caucasian, 6.0\% African American, and 7.9\% other races.

Given that the final sample was somewhat younger and better educated than the original sample, we must take these limitations into account when considering the generalizability of the findings (Radler \& Ryff, 2010). Table 1 presents demographic and health data for participants grouped into five age groups: one (32-44 years), two (45-54), three (55-64), four (65-74), and five (7584). Mean educational levels for the groups ranged from 13.45 to 14.78 years.

\section{Measures}

Demographics and health. Measures included sex, highest level of education in years, and household income. Health measures (rated on a 5-point scale from 1 [excellent] to 5 [poor]) included self-rated health status, vision, hearing, and limitations in activities of daily living and instrumental activities of daily living (Lawton \& Brody, 1969).

Computer use questions. Participants rated how often they used a computer (e.g., for e-mail, Internet searching, etc.) on a 
Table 1

Participant Characteristics and BTACT Scores

\begin{tabular}{|c|c|c|c|c|c|c|c|c|c|c|}
\hline \multirow[b]{2}{*}{ Variable } & \multicolumn{2}{|c|}{ Age $32-44$} & \multicolumn{2}{|c|}{ Age $45-54$} & \multicolumn{2}{|c|}{ Age 55-64 } & \multicolumn{2}{|c|}{ Age $65-74$} & \multicolumn{2}{|c|}{ Age $75-84$} \\
\hline & $M$ & $S D$ & $M$ & $S D$ & $M$ & $S D$ & $M$ & $S D$ & $M$ & $S D$ \\
\hline$n$ & \multicolumn{2}{|c|}{568} & \multicolumn{2}{|c|}{754} & \multicolumn{2}{|c|}{714} & \multicolumn{2}{|c|}{451} & \multicolumn{2}{|c|}{184} \\
\hline Age & 39.77 & 3.14 & 49.56 & 2.91 & 59.11 & 2.90 & 68.86 & 2.87 & 78.36 & 2.45 \\
\hline Sex ( $\%$ female) & \multicolumn{2}{|c|}{60.0} & \multicolumn{2}{|c|}{51.9} & \multirow{2}{*}{\multicolumn{2}{|c|}{$\begin{array}{l}51.5 \\
40.8\end{array}$}} & \multirow{2}{*}{\multicolumn{2}{|c|}{$\begin{array}{l}58.5 \\
32\end{array}$}} & \multicolumn{2}{|c|}{56.0} \\
\hline Education $^{\mathrm{a}}(\%)$ & \multicolumn{2}{|c|}{47.2} & \multicolumn{2}{|c|}{44.0} & & & & & \multicolumn{2}{|c|}{28.3} \\
\hline Income $^{\mathrm{b}}$ & 89.0 & 55.9 & 94.8 & 55.7 & 84.2 & 57.2 & 57.2 & 39.1 & 47.9 & 44.7 \\
\hline Health $^{c}$ & 3.83 & 0.88 & 3.73 & 0.91 & 3.71 & 0.97 & 3.63 & 0.93 & 3.34 & 0.94 \\
\hline Hearing $^{c}$ & 3.70 & 0.98 & 3.42 & 1.04 & 3.46 & 1.08 & 3.41 & 1.05 & 3.04 & 1.12 \\
\hline Vision $^{\mathrm{c}}$ & 3.41 & 1.08 & 3.09 & 0.94 & 3.33 & 0.88 & 3.41 & 0.87 & 3.40 & 0.95 \\
\hline Limitations $^{\mathrm{d}}$ & 2.45 & 4.36 & 4.06 & 5.61 & 5.18 & 6.29 & 7.55 & 7.11 & 10.37 & 7.00 \\
\hline Computer activity days/week & 5.05 & 2.66 & 4.90 & 2.85 & 4.37 & 3.04 & 3.25 & 3.22 & 1.64 & 2.77 \\
\hline BTACT composite score & 0.38 & 0.62 & 0.20 & 0.60 & 0.01 & 0.62 & -0.25 & 0.61 & -0.57 & 0.55 \\
\hline SGST: Task-switching latencies ${ }^{\mathrm{e}}$ & 0.98 & 0.18 & 1.02 & 0.18 & 1.06 & 0.20 & 1.14 & 0.23 & 1.18 & 0.23 \\
\hline SGST: Single-task baseline latencies ${ }^{f}$ & 0.77 & 0.13 & 0.79 & 0.12 & 0.83 & 0.13 & 0.88 & 0.14 & 0.93 & 0.15 \\
\hline
\end{tabular}

Note. $\quad$ BTACT $=$ Brief Test of Adult Cognition by Telephone; SGST $=$ Stop and Go Switch Task

${ }^{\mathrm{a}} \%$ with 4-year college degree and above. ${ }^{\mathrm{b}}$ Income reported in $\$ 1,000 \mathrm{~s} .{ }^{\mathrm{c}}$ Rated on a scale from 1 (poor) to 5 (excellent). ${ }^{\mathrm{d}}$ Number of limitations (activities of daily living plus instrumental activities of daily living). ${ }^{\mathrm{e}}$ Reaction time in seconds on task switching (mean of switch and nonswitch trials). $\quad{ }^{\mathrm{f}}$ Reaction time in seconds on single-task baseline ("normal" condition).

6-point scale from 1 (never) to 6 (daily). Scores were recoded into activity days per week (Verghese et al., 2003), so that using the computer at the following frequencies earned participants points: daily, 7 points; several times a week, 4 points; once a week, 1 point; several times a month, 0.5 points; once a month, 0.25 points; and never, 0 points. Previous research has shown good test-retest reliability for similar rating scales (Hultsch et al., 1999; Verghese et al., 2003). In addition, we asked how frequently participants used the computer on the job, using a 5-point scale $(1=$ all of the time, $2=$ most of the time, $3=$ some of the time, $4=$ little of the time, and $5=$ never .

Cognitive performance. Cognition was assessed in telephone interviews using the BTACT battery (Lachman \& Tun, 2008; Tun \& Lachman, 2005; Tun \& Lachman, 2006), that assesses the following key cognitive domains: episodic verbal memory: summed immediate and delayed recall of a 15-word list (after Rey, 1964); working memory span: backward digit span (Wechsler, 1997); executive function: category fluency (lezak, 1995); inductive reasoning: number series completion; speed of processing: backward counting for $30 \mathrm{~s}$. A live interviewer administered the tests over the phone in a fixed order, and the participant responded verbally; the battery averaged 15-20 min and was designed to not fatigue our participants.

A BTACT composite score was created from standardized accuracy scores on these subtests, based on an exploratory factor analysis (Lachman \& Tun, 2008). A principal axis factor analysis with oblique rotation yielded one factor with an eigenvalue greater than one (see Table 2). A factor score, labeled the BTACT Composite, was computed by averaging the standardized values of each variable and then standardizing that mean score. The factor accounts for $34 \%$ of the total variance, with a moderate alpha reliability of .55 .

Task-switching latencies. In addition, reaction time and taskswitching were measured with the SGST (Tun \& Lachman, 2008), a speeded test that included two single-task blocks and a mixed task-switching block that required alternating between two tasks, with substantial involvement of central executive function (Baddeley, 2002). Criterion for inclusion of cases was $75 \%$ accuracy on all conditions. Measures were latencies on correct trials for the single-task and the task-switching test (mean of switch and nonswitch trials).

Correlations of task-switching with BTACT tests and executive function tests. Table 3 presents intercorrelations between latencies on the simple baseline and the task-switching tests of the SGST, with BTACT tests given over the telephone (Lachman, Tun, Agrigoraei, \& Murphy, 2009). In addition, we present correlations of the SGST and BTACT measures with standard neuropsychological tests that were administered in person to a Boston area subsample of the MIDUS that included 151 longitudinal participants and an additional 148 participants recruited at testing Time 2. We recognize that the cognitive battery designed for large scale survey use is limited in its ability to operationalize key cognitive constructs such as fluid intelligence, speed of processing, working memory, and executive function with only one indicator of each dimension (Salthouse, 2009; Verhaeghen \& Salthouse, 1997). Nevertheless, we have some evidence for convergent validity with other standard tests of these cognitive dimensions (Lachman et al., 2009).

Table 2

Factor Matrix for Principal Axis Exploratory Factor Analysis for BTACT Measures

\begin{tabular}{lc}
\hline \multicolumn{1}{c}{ BTACT test } & Factor $1^{\mathrm{a}}$ \\
\hline Number Series & .68 \\
Backward Counting & .67 \\
Category Fluency & .56 \\
Digits Backward & .48 \\
Word List Composite (sum of Word Lists & .48 \\
\hline Immediate and Delayed) & \\
\hline
\end{tabular}

Note. $\quad$ BTACT $=$ Brief Test of Adult Cognition by Telephone.

${ }^{\mathrm{a}}$ Eigenvalue. 
Table 3

Intercorrelations of SGST Measures With BTACT and In-Person Neuropsychological Tests

\begin{tabular}{|c|c|c|c|c|c|c|c|c|}
\hline Variable & $\begin{array}{l}\text { SGST: Single- } \\
\text { task baseline }\end{array}$ & $\begin{array}{l}\text { SGST Task } \\
\text { Switching }\end{array}$ & $\begin{array}{l}\text { Word list recall: } \\
\text { Immediate }\end{array}$ & $\begin{array}{l}\text { Word list } \\
\text { recall: Delayed }\end{array}$ & $\begin{array}{l}\text { Backward } \\
\text { Digit Span }\end{array}$ & $\begin{array}{l}\text { Verbal } \\
\text { Fluency }\end{array}$ & $\begin{array}{l}\text { Number } \\
\text { Series }\end{array}$ & $\begin{array}{l}\text { Backward } \\
\text { Counting }\end{array}$ \\
\hline \multicolumn{9}{|c|}{ BTACT measure } \\
\hline SGST Task Switching & $.72(2671)$ & & & & & & & \\
\hline Word list recall: Immediate & $-.25(2671)$ & $-.20(2671)$ & & & & & & \\
\hline Word list recall: Delayed & $-.23(2671)$ & $-.19(2671)$ & $.79(2671)$ & & & & & \\
\hline Backward Digit Span & $-.24(2671)$ & $-.18(2671)$ & $.36(2671)$ & $.33(2671)$ & & & & \\
\hline Verbal Fluency & $-.28(2671)$ & $-.31(2671)$ & $.25(2671)$ & $.24(2671)$ & $.20(2671)$ & & & \\
\hline Number Series & $-.27(2671)$ & $-.29(2671)$ & $.26(2671)$ & $.25(2671)$ & $.33(2671)$ & & & \\
\hline Backward Counting & $-.43(2671)$ & $-.46(2671)$ & $.24(2671)$ & $.22(2671)$ & $.29(2671)$ & $.39(2671)$ & $.45(2671)$ & \\
\hline \multicolumn{9}{|c|}{ In-person tests } \\
\hline Digit Symbol Substitution & $-.46(239)$ & $-.47(239)$ & $.38(252)$ & $.38(247)$ & $.29(251)$ & $.42(252)$ & $.38(251)$ & $.51(252)$ \\
\hline Trail Making A & $.28(227)$ & $.32(227)$ & $-.20^{* *}(239)$ & $-.24(234)$ & $-.22(238)$ & $-.27(239)$ & $-.32(238)$ & $-.34(239)$ \\
\hline Trail Making B & $.34(212)$ & $.43(212)$ & $-.29(224)$ & $-.30(219)$ & $-.33(223)$ & $-.34(224)$ & $-.40(223)$ & $-.41(224)$ \\
\hline Letter Number Sequencing & $-.40(216)$ & $-.46(216)$ & $.26(228)$ & $.20^{* *}(223)$ & $.39(228)$ & $.32(228)$ & $.45(227)$ & $.43(228)$ \\
\hline
\end{tabular}

Note. The values in parentheses represent the number of participants for each combination of measures. BTACT $=$ Brief Test of Adult Cognition by Telephone; SGST $=$ Stop and Go Switch Task. All correlations significant at $p<.001$, unless noted as ${ }^{* *}(p<.01)$.

The correlations of the SGST latencies with BTACT tests are modest for the measures of memory and tasks that draw on fluid abilities (Category Fluency and Number Series Completion), and largest for the speeded backward counting task. This suggests that the SGST assesses some nonoverlapping abilities, but that the SGST latencies and the other BTACT tests share a reliance on processing speed; this is logical because the auditory presentation of the tests requires online processing in real time, without review, and thus involves an element of speeded performance. However, the correlations of the task-switching latencies are stronger for the in-person neuropsychological tests considered to represent the gold standard of executive function measures, such as Trailmaking (Reitan, 1958) and Digit Symbol Substitution (Wechsler, 1997). This provides convergent validity for the task-switch latencies as a measure of executive function.

\section{Results}

\section{Patterns of Computer Activity Across Age Groups}

Table 4 shows a good range of frequency of computer use within each age group. The majority in the younger groups were daily computer users, but older groups also showed substantial computer use, with about $36 \%$ reporting some experience even in the oldest group. These levels of activity are somewhat lower than those reported by Czaja et al. (2006) for a group of well-educated volunteers, but somewhat higher than large-scale surveys of Internet use: For age 30-49, our data shows 92\% computer users compared to $86 \%$ reported by Fox (2006), and for age 50-64, our data shows $83 \%$ compared to their $70 \%$. Computer use was reported by $60 \%$ of our participants in the $65-74$ group, and $36 \%$ in the 75-85 group, compared to the Fox (2006) report of about $33 \%$ in the $65+$ group.

Differences in computer use by age, sex, and education. An analysis of covariance on frequency of computer use included age (5), sex (2), and education (2: less than college degree, college degree or higher; preliminary analyses showed that this division captured important differences) as between-subjects variables. Household income; race; self-ratings of overall health, vision, and hearing; and limitations were covariates. Computer use was positively related to self-rated health, $F(1,2645)=5.15, p<.05$, $\eta_{\mathrm{p}}^{2}=.002$, and income, $F(1,2645)=57.23, p<.001, \eta_{\mathrm{p}}^{2}=.021$, but race was not significant, $F(1,2645)<1.0$. Computer use was

Table 4

Frequency of Computer Activity: Percentage by Age Group

\begin{tabular}{lrrrrr}
\hline & \multicolumn{5}{c}{ Age group } \\
\cline { 2 - 6 } \multicolumn{1}{c}{$\begin{array}{c}\text { Frequency of } \\
\text { computer use }\end{array}$} & $32-44$ & $45-54$ & $55-64$ & $65-74$ & $75-85$ \\
\hline Never & 6.2 & 11.5 & 19.0 & 39.5 & 63.6 \\
Monthly & 5.5 & 3.3 & 3.1 & 4.0 & 3.3 \\
Several times per month & 5.6 & 6.0 & 5.9 & 2.4 & 4.9 \\
Weekly & 3.3 & 4.4 & 4.3 & 2.9 & 3.3 \\
Several times per week & 19.4 & 14.1 & 14.7 & 12.9 & 6.0 \\
Daily & 60.0 & 60.7 & 52.9 & 39.4 & 19.0 \\
Total \% & 100 & 100 & 100 & 100 & 100 \\
Total $N$ & 568 & 754 & 714 & 451 & 184 \\
\hline
\end{tabular}


also positively related to self-rated vision, $F(1,2645)=3.95, p<$ $.05, \eta_{\mathrm{p}}^{2}=.001$, but not to self-rated hearing or limitations $(p>$ $.05)$. Consistent with previous studies, greater computer activity was associated with younger age, $F(4,2645)=34.78, p<.001$, $\eta_{\mathrm{p}}^{2}=.050$, and higher education levels, $F(1,2645)=113.24, p<$ $.001, \eta_{\mathrm{p}}^{2}=.041$ (Czaja et al., 2006; Fox, 2001). Bonferroni comparisons showed no significant age difference in use between the two younger groups but drops in rates of use for age 55-64, with further drops for age $65-74$ and for age 75-85. We found no overall difference by sex, confirming Czaja et al. (2006), nor an Age $\times$ Sex interaction. The interaction of sex and education was significant, $F(1,2645)=27.88, p<.001, \eta_{\mathrm{p}}^{2}=.010$; women used the computer more than men in the lower education group, but men used the computer more than women in the higher education group. However, this interaction was nonsignificant after controlling for frequency of using the computer on the job (as a covariate) in the under-65 groups $(N=2,036)$, the group with the large majority still in the workforce (Charness \& Boot, 2009). Thus, the interaction appears to be driven by on-the-job computer use by younger, employed participants. In the under-65 group with lower education, only $31.1 \%$ of the men use the computer regularly in their job, versus $55.3 \%$ of the women; among the higher educated, $68.5 \%$ of the men and $57.0 \%$ of the women report regular use of the computer on the job.

\section{Frequency of Computer Use and Cognitive Performance}

We next examined the association between computer use and performance on cognitive tasks. Table 1 gives cognitive scores for five age groups on the BTACT composite and SGST.

Regression analyses: Level of computer activity and cognitive performance. We investigated the association between computer activity and cognitive performance, using hierarchal regression analyses to examine the effect of frequency of computer use on the BTACT composite score (see Table 5). The order of entry was forced and was as follows: Age, sex, income, education, and health status (self-rated health and physical limitations), all of which can impact cognitive performance (e.g., Hultsch, Hammer, $\&$ Small, 1993) were entered before computer activity (days of activity per week) to determine whether computer activity contributes additional variance to cognitive performance. Next we entered two-way interactions between age, computer use, and the predictors of interest, followed by three-way interactions among those variables. Our goal was to determine how much variance could be explained, after age and the other independent variables, by computer activity and its interactions. Higher order interactions were retained in the model on the basis of the significance of the $F$ statistic for increments to $R^{2}$. The model was trimmed to include only significant predictors and interactions; income and physical limitations did not make a significant contribution to the model, and were dropped in the following regression analysis.

Table 5 shows that age had a robust negative association with the BTACT composite score, accounting for about $17 \%$ of the variance. Higher levels of education and self-rated health were associated with better performance on all measures, but sex was nonsignificant. Most importantly, higher levels of computer activity made a modest but significant contribution to the BTACT composite $(p<.001)$, reflecting better cognitive performance for participants who used the computer frequently. Adults who used the computer daily scored about two-thirds of a standard deviation higher on the BTACT than those who used the computer only once a month. The effect of each increment in frequency of computer use (representing one day per week of activity) was similar to that seen for increments in ratings of health (fair to good) or education (e.g., high school to 1-2 years of college).

A significant interaction of Sex $\times$ Computer Use $(p<.01)$ showed that the effect of computer use was larger for men than for women; indeed, men with low levels of computer use had the lowest scores of any of the groups. However, the interaction between age and computer use was not significant, indicating that the association between computer use and BTACT performance was equivalent across adulthood.

Secondary analyses excluding participants with possible memory impairment. To address the concern that the association between computer use and cognitive performance in the oldest groups might be biased by individuals with early signs of dementia who restrict their computer activity, we performed secondary analyses excluding older adults with especially poor episodic memory, an early indication of Alzheimer's disease. Excluding the lowest $10 \%$ of episodic memory scores from the two oldest age groups did not change the pattern of results in regression analyses, indicating that our findings were not due to the presence of impaired participants.

\section{Switch-Task Latencies: Computer Activity and Executive Function}

Although the previous regression analysis suggests that greater computer activity is associated with generally better cognitive

Table 5

Hierarchal Regression Analyses of Computer Activity Predicting Performance on BTACT Composite Score Adjusting for Sex, Education, and Health Status

\begin{tabular}{|c|c|c|c|c|c|c|}
\hline Significant predictor & $\beta$ & Adjusted $R^{2}$ & Increment to $R^{2}$ & Increment to $F$ & $d f_{\mathrm{s}}$ & $p$ \\
\hline Age & -.313 & .172 & .172 & 555.35 & 1,2669 & .000 \\
\hline Sex & .019 & .172 & .000 & 0.79 & 1,2668 & $n s$ \\
\hline Education & .291 & .308 & .135 & 520.83 & 1,2667 & .000 \\
\hline Health & .105 & .319 & .013 & 49.66 & 1,2666 & .000 \\
\hline Computer activity & .286 & .339 & .019 & 78.34 & 1,2665 & .000 \\
\hline Sex $\times$ Computer Activity & -.135 & .340 & .002 & 6.91 & 1,2664 & .009 \\
\hline
\end{tabular}

Note. Beta values are standardized coefficients associated with the model. BTACT $=$ Brief Test of Adult Cognition by Telephone. 
performance, we next investigated whether computer activity is associated with better performance in specific domains such as executive function by examining the association of computer use with response latencies on the SGST, a speeded task-switching test. In addition to demographic and health variables, we controlled for basic cognitive ability (using the BTACT composite score) before entering computer use as a predictor, thus performing a conservative test of whether computer activity makes an additional contribution to task-switch latencies in individuals of similar mental ability. Although the BTACT subtests that go into the composite score involve some executive processing, the pattern of correlations shown in Table 4 would suggest that task-switching requires nonoverlapping skills. For example, although the verbal fluency measure of the BTACT involves executive functions, it also provides an index of verbal ability, an important crystallized intelligence measure (Tombaugh, Kozak, \& Rees, 1999).

Although hierarchical regression models with cross-sectional data cannot provide definitive answers about causal relations or mediation (Lindenberger \& Potter, 1998), this approach provides preliminary supportive and informative evidence for relationships that should be pursued in future research using other designs. We reasoned that if computer use contributed to task switching after controlling for the BTACT composite, this would suggest a domain-specific association between computer use and executive control functions such as task switching and inhibitory control.

Hierarchal regressions on reaction times for both single-task and task-switching trials used a forced-entry method in which age, sex, education level, and health status were entered, followed by basic cognitive ability (scores on the BTACT composite), and then by frequency of computer activity. We next entered two-way interactions between age, computer activity, and the predictors of interest, followed by three-way interactions. Our goal was to determine how much variance could be accounted for by computer activity after accounting for age, demographics, health, and basic cognitive ability. As in the previous analyses, the model was trimmed to include only significant predictors and interactions; income and physical limitations did not make a significant contribution to the model, and were dropped in the following analysis.
Table 6 shows that younger age, male sex, higher education, and better health were positively associated with task-switching performance, as were higher BTACT scores, as expected. Most importantly, computer activity made a significant contribution to task-switching performance, after accounting for basic cognitive ability. The significant interaction between computer use and cognitive ability shown in Figure 1 indicates that differences in task switching associated with computer activity were greater for those of lower cognitive ability. That is, the difference in task switching between high use and low use of the computer was greatest in the group with low cognitive scores. This suggests that higher levels of computer activity are associated with a greater increase in executive function for those with relatively low cognitive ability. As predicted, computer activity did not make a significant contribution to variance in single-task latencies that involve mainly speed of processing, after controlling for basic cognitive ability.

Overall, these findings indicate that frequency of computer use makes a significant contribution to speeded task-switching performance, even when individuals are equated for basic cognitive function in domains of fluid intelligence as well as age, education, and health status.

\section{Discussion}

Our findings offer new insight into the association between computer activity and cognition across the lifespan in adults from a large national sample with wide range of age, education, and socioeconomic backgrounds. These data demonstrate that frequent computer use is associated with better overall cognitive performance across adulthood, from younger adults through middle-aged and older adults. Also, we found a positive association between computer use and executive function that was seen even after controlling for basic intellectual ability. Specifically, more frequent computer use was associated with better task-switching performance, and this association was strongest in adults with lower general cognitive ability.

Table 6

Hierarchal Regression Analyses With Response Latencies on Task-Switching and a Choice Reaction Time Test Regressed on Computer Activity, Adjusting for Age, Sex, Education, Health Status, and Basic Cognitive Ability

\begin{tabular}{|c|c|c|c|c|c|c|}
\hline Significant predictors & $\beta$ & Adjusted $R^{2}$ & Increment to $R^{2}$ & Increment to $F$ & $d f \mathrm{~s}$ & $p$ \\
\hline \multicolumn{7}{|l|}{ Task-switching latencies } \\
\hline Age & -.010 & .095 & .096 & 282.29 & 1,2669 & .000 \\
\hline Sex & .089 & .103 & .008 & 22.98 & 1,2668 & .000 \\
\hline Education & .029 & .120 & .018 & 54.83 & 1,2667 & .000 \\
\hline Health & -.053 & .129 & .009 & 28.25 & 1,2666 & .000 \\
\hline Basic cognitive ability ${ }^{a}$ & -.336 & .211 & .082 & 297.90 & 1,2665 & .000 \\
\hline Computer activity & -.059 & .215 & .004 & 13.20 & 1,2664 & .000 \\
\hline Age $\times \operatorname{Sex}$ & .159 & .217 & .003 & 8.91 & 1,2663 & .003 \\
\hline Computer Activity $\times$ Cognitive Ability & .070 & .221 & .007 & 12.34 & 1,2662 & .000 \\
\hline \multicolumn{7}{|l|}{ Baseline latencies } \\
\hline Age & .222 & .129 & .130 & 397.33 & 1,2669 & .000 \\
\hline Education & .049 & .136 & .008 & 23.41 & 1,2667 & .000 \\
\hline Health & -.036 & .141 & .005 & 16.24 & 1,2666 & .000 \\
\hline Basic cognitive ability ${ }^{\mathrm{a}}$ & -.319 & .213 & .072 & 242.60 & 1,2665 & .000 \\
\hline
\end{tabular}

Note. Beta values are standardized coefficients associated with the model. BTACT $=$ Brief Test of Adult Cognition by Telephone

${ }^{a}$ BTACT composite score. 


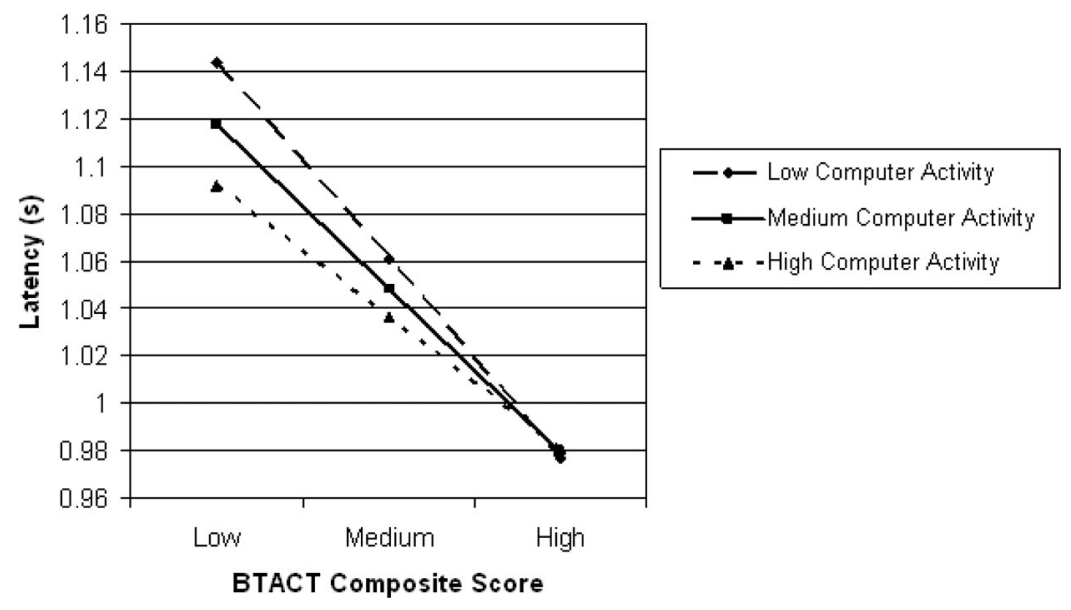

Figure 1. Task-switching latencies for groups with low, medium and high cognitive scores (BTACT composite) by frequency of computer activity (low $[-1 S D]$, medium $[M]$, high $[+1 S D]$ ). BTACT $=$ Brief Test of Adult Cognition by Telephone.

The work presented here represents the first large sample $(N=$ 2,671 ) drawn from a national, population-based study to report a positive association between frequency of computer use and cognitive performance from young adulthood into old age. Our findings demonstrate that computer activity is associated with higher levels of performance on cognitive tasks even after controlling for effects of age, sex, education, and health status. Although one cannot determine the directionality of the association between computer use and cognitive performance with cross-sectional data, the scope of the MIDUS national sample allows us to affirm that regular computer use was associated with better performance on the BTACT (Tun \& Lachman, 2006), a cognitive battery that included tests of episodic memory and working memory, reasoning, and processing speed. We found that individuals who used the computer daily scored about two-thirds of a standard deviation higher on the BTACT than those who used the computer only once a month. Each day of computer activity per week was associated with increments in cognitive performance similar to those seen for higher levels of health or a few years of education. These effects were consistent from young adulthood across middle age and old age.

In an additional set of analyses, we found evidence for a domain-specific association: More frequent computer use was associated with better executive function (i.e., greater speed in task switching) but not simple speed of processing, even after controlling for basic cognitive abilities. This is consistent with a recent imaging study of older adults showing that greater computer expertise was associated with higher levels of activation in brain regions associated with decision making during a search task (Small, Moody, Siddarth, \& Bookheimer, 2009). Also, neuroimaging work has demonstrated that task-switching tests can involve significant frontal lobe activity, particularly in older adults, (Smith et al., 2001), and extensive training in use of a video game improved performance by older adults on some cognitive tasks, with a selective advantage for task switching (Basak et al., 2008).

Moreover, we found that the association between computer use and task switching was greater for those with lower basic cognitive ability compared to those with higher basic cognitive ability (see
Salthouse, 2006); those with lower levels of cognitive ability who regularly used the computer showed differentially better executive function in task switching than participants of similar basic ability who seldom used the computer. These findings demonstrate that frequent computer activity is associated with better executive control function in individuals with fewer intellectual and educational advantages.

Our findings for patterns of computer use also extend previous research (e.g., Czaja et al., 2006) by including a more diverse national sample with a wider range of age, education, and socioeconomic backgrounds. Although use of the computer declined across age groups, as expected (Czaja et al., 2006), the majority of our participants (58\%) aged 60 and over reported using the computer. Greater use of the computer was associated with higher levels of education, income, and health, consistent with previous reports (Czaja et al., 2006). We also found that in groups with lower education levels, women used the computer more than men, while among those with college degrees, men used the computer more than women; these sex differences appear to be driven mainly by job-related use of the computer by younger adults who are employed. Interestingly, however, we also found that the disparity in cognitive scores between regular computer users and nonusers was larger for men than for women. Together, these findings raise a concern for men, in particular, that poor cognitive function may be associated with falling behind in technology use. Again, we cannot determine the direction of this association, and it is possible that having and maintaining a job that involves a computer also requires more mental activities such as organizing and multitasking.

Nevertheless, we cautiously interpret these findings as suggesting that the positive association between frequency of computer use and executive function is not due merely to self-selection of brighter, fitter individuals using the computer. Rather, our results may be seen as consistent with the notion that engaging in a mentally challenging activity such as using the computer can support cognitive function across adulthood (cf. Hultsch et al., 1999; Schooler, 2007; Wilson et al., 1999). In addition, the finding that the association between computer user and executive function 
was greater for those with lower basic cognitive ability, compared to those with higher ability, allows us to speculate that computer activity may be especially important for those who are cognitively disadvantaged. This echoes the concern that in an increasingly technology-based society the futures of technological "have-nots" may be limited if they become increasingly disenfranchised.

The next step is to specify aspects of computer use that have the strongest relationship with particular aspects of cognitive performance. Recent research has shown no cognitive benefits for older adults in using the Internet for a period of 1 year (Slegers et al., 2009). However, just as physical activity has been associated with better performance in specific cognitive domains such as executive function (e.g., Hillman, Erickson, \& Kramer, 2008), computer use may have a specific association with executive functions. Neuroimaging shows that older adults recruit areas of prefrontal cortex during executive function tasks (Smith et al., 2001) and that older adults with greater computer experience show greater brain activation in frontal regions during an Internet searching task (Small et al., 2009). Moreover, findings that computer experience is associated with altered neural activation in frontal regions (Haier et al., 1992) are consistent with the computer's demands on multitasking, involving shifting of attention and interactive coordination of motor, sensory, and cognitive skills. Thus, one might further speculate that coordinating and scheduling all of these component activities may help maintain good cognitive function in adulthood, much like the putative effects of education (Stern et al., 2005) and mentally challenging occupations (Schooler, 2007).

It is also true that frequency of computer use will depend on noncognitive factors such as interest and belief in computer utility, as well as computer anxiety and efficacy (Czaja et al., 2006; Umemuro, 2004). Also, proposed noncognitive benefits of computer use include supporting older individuals' autonomy, and contributing to well-being and sense of empowerment (Shapira, Barak, \& Gal, 2007), although a recent randomized trial found no such benefit (Slegers, van Boxtel, \& Jolles, 2008). A challenge for future research is to elucidate these effects further.

\section{Conclusions: Limitations and Future Directions}

A limitation of these findings is that they are based on crosssectional observations, with self-reported ratings of health and computer activity. Although we cannot claim that this sample is strictly representative of the population, as there is inevitably some shift toward retention of younger and better educated participants, this represents the first large national study to address these issues, and it includes a wider range of age, educational, and geographic backgrounds than has been previously sampled. Also, although participants reported how often they used the computer, and the extent of job-related computer use, more detailed information on how the computer is used, and for what duration would be useful; we plan to address this in future work.

Although computer use may be just one of many forms of activity that are associated with good mental function, a reasonable goal is to make computers available to all sectors of the population. Nationally, increasing numbers of older adults rely on the Internet for communication, research, and banking (Fox, 2006), as well as innovative applications such as exercise plans (Kressig \& Echt, 2002) and support groups (Marziali \& Donahue, 2006). However, our study confirmed that those with lower income and education are less likely to use computers, particularly among older adults (Fox, 2006), and so it is important to provide public access, perhaps through federal programs such as Neighborhood Networks (U.S. Department of Housing and Urban Development, 2008). In addition, the low level of computer use found for less educated men in this study raises the concern that in an increasingly technology-based society the "digital divide" will become more pronounced. Our findings suggest that it is important that adults of all ages have access to computers through public programs in community centers, libraries, and senior centers. Age-appropriate instruction should address the special challenges computers present for older persons including psychomotor and sensory issues, pacing, dividing attention, and lack of face-to-face interaction (Willis, 2006). Future technological advances and lower costs may reduce some of the barriers that discourage some adults from using computer technology (Charness \& Boot, 2009).

In summary, our findings suggest that frequent computer use is associated with significantly better cognitive performance across adulthood. Moreover, regular computer use is associated with better executive function through middle age and later life, even for adults who are similar in basic intellectual ability as well as education and health status. Future research will further delineate the nature of this association, and help determine whether specific aspects of computer activity might be associated with better performance in specific cognitive domains.

\section{References}

Baddeley, A. (2002). Fractionating the central executive. In D. T. Stuss \& R. T. Knight (Eds.), Principles of frontal lobe function (pp. 246-277). New York, NY: Oxford University Press.

Basak, C., Boot, W. R., Voss, M. W., \& Kramer, A. F. (2008). Can training in a real-time strategy video game attenuate cognitive decline in older adults? Psychology and Aging, 23, 765-777. doi:10.1037/a0013494

Brim, O., Ryff, C., \& Kessler, R. (2004). How healthy are we? A national study of well-being at midlife. Chicago, IL: University of Chicago Press.

Charness, N., \& Boot, W. R. (2009). Aging and information technology use: Potential and barriers. Current Directions in Psychological Science, 18, 253-258. doi:10.1111/j.1467-8721.2009.01647.x

Czaja, S. J., Charness, N., Fisk, A. D., Hertzog, C., Nair, S. N., Rogers, W. A., \& Sharit, J. (2006). Factors predicting the use of technology: Findings from the Center for Research on Aging and Technology Enhancement (CREATE). Psychology and Aging, 21, 333-352. doi: 10.1037/0882-7974.21.2.333

Fox, S. (2001). Wired seniors: A fervent few, inspired by family ties. Washington, DC: Pew Foundation.

Fox, S. (2006). Seniors and the Internet. Retrieved from http:// www.pewinternet.org/Presentations/2006/Seniors-and-the-Internet.aspx

Fritsch, T., McClendon, M. J., Smyth, K. A., Lerner, A. J., Friedland, R. P., \& Larsen, J. D. (2007). Cognitive functioning in healthy aging: The role of reserve and lifestyle factors early in life. Gerontologist, 47, 307-322.

Haier, R. J., Siegel, B. V., MacLachlan, A., Soederling, E., Lottenberg, S., \& Buchsbaum, M. S. (1992). Regional glucose metabolic changes after learning a complex visuospatial/motor task. Brain Research, 570, 134 143. doi:10.1016/0006-8993(92)90573-R

Hillman, C. H., Erickson, K. I., \& Kramer, A. F. (2008). Be smart, exercise your heart: Exercise effects on brain and cognition. Nature Reviews Neuroscience, 9, 58-65. doi:10.1038/nrn2298

Hultsch, D. F., Hammer, M., \& Small, B. J. (1993). Age differences in cognitive performance in later life: Relationships to self-reported health and activity life style. Journals of Gerontology, Series B: Psychological Sciences and Social Sciences, 48, 1-11. 
Hultsch, D. F., Hertzog, C., Small, B. J., \& Dixon, R. A. (1999). Use it or lose it: Engaged lifestyle as a buffer of cognitive decline in aging? Psychology and Aging, 14, 245-263. doi:10.1037/0882-7974.14.2.245

Kane, R. L., Boston, K., \& Chilvers, M. (2007). Helping people make better long-term-care decisions. Gerontologist, 47, 244-247.

Kressig, R. W., \& Echt, K. V. (2002). Exercise prescribing: Computer application in older adults. Gerontologist, 42, 273-277.

Lachman, M. E., Agrigoraei, S., Murphy, C., \& Tun, P. (2010). Frequent cognitive activity compensates for education differences in episodic memory. American Journal of Geriatric Psychiatry, 18, 4-10. doi: 10.1097/JGP.0b013e3181ab8b62

Lachman, M. E., \& Tun, P. A. (2008). Cognitive testing in large-scale surveys: Assessment by telephone. In D. Alwin \& S. Hofer (Eds.), Handbook on cognitive aging: interdisciplinary perspectives (pp. 506523). New York, NY: Sage.

Lachman, M. E., Tun, P. A., Agrigoraei, S., \& Murphy, C. (2009). Cognition in midlife: Findings from the Brief Test of Adult Cognition by Telephone (BTACT) with the MIDUS National Sample. Unpublished manuscript, Brandeis University.

Lawton, M. P., \& Brody, E. M. (1969). Assessment of older people: Self maintaining and instrumental activities of daily living. Gerontologist, 9 , 179-186.

Lezak, M. D. (1995). Neuropsychological assessment (3rd ed.). New York, NY: Oxford University Press.

Lindenberger, U., \& Potter, U. (1998). The complex nature of unique and shared effects in hierarchal linear regression: Implications for developmental psychology. Psychological Methods, 32, 218-230.

Marziali, E., \& Donahue, P. (2006). Caring for others: Internet videoconferencing group intervention for family caregivers of older adults with neurodegenerative diseases. Gerontologist, 46, 398-403.

Ownby, R. L., Czaja, S. J., Loewenstein, D., \& Rubert, M. (2008). Cognitive abilities that predict success in a computer-based training program. Gerontologist, 48, 170-180.

Radler, B. T., \& Ryff, C. D. (2010). Who participates? Accounting for longitudinal retention in the MIDUS (Midlife in the U.S.) National Study of Health and Well-Being. Journal of Aging \& Health, 22, 307-331.

Reitan, R. M. (1958). Validity of the Trail Making Test as an indication of organic brain damage. Perceptual and Motor Skills, 8, 271-276. doi: 10.2466/PMS.8.7.271-276

Rey, A. (1964). L'examen clinique en psychologie [The Clinical Examination in Psychology]. Paris, France: Presses Universitaires de France.

Saczynski, J. S., Jonsdottir, M. K., Sigurdsson, S., Eiriksdottir, G., Jonsson, P. V., Garcia, M. E., . . Launer, L. J. (2008). White matter lesions and cognitive performance: The role of cognitively complex leisure activity. Journals of Gerontology, Series A: Biological Sciences and Medical Sciences, 63, 848-854.

Salthouse, T. A. (2006). Mental exercise and mental aging. Perspectives on Psychological Science, 1, 68-87. doi:10.1111/j.1745-6916.2006.00005.x

Salthouse, T. A. (2009). Decomposing age correlations on neuropsychological and cognitive variables. Journal of the International Neuropsychological Society, 15, 650-661. doi:10.1017/S1355617709990385

Schooler, C. (2007). Use it—and keep it longer, probably. Perspectives on Psychological Science, 2, 24-29. doi:10.1111/j.1745-6916.2007.00026.x

Shapira, N., Barak, A., \& Gal, I. (2007). Promoting older adults' wellbeing through Internet training and use. Aging \& Mental Health, 11, 477-484. doi:10.1080/13607860601086546

Slegers, K., van Boxtel, M. P. J., \& Jolles, J. (2008). Effects of computer training and Internet usage on the well-being and quality of life of older adults: A randomized, controlled study. Journals of Gerontology, Series B: Psychological Sciences and Social Sciences, 6, 176-184.
Slegers, K., van Boxtel, M. P. J., \& Jolles, J. (2009). Effects of computer training and Internet usage on cognitive abilities in older adults: A randomized controlled study. Aging, Clinical and Experimental Research, 21, 43-54.

Small, G. W., Moody, T. D., Siddarth, P., \& Bookheimer, S. Y. (2009). Your brain on Google: Patterns of cerebral activation during internet searching. American Journal of Geriatric Psychiatry, 17, 116-126. doi:10.1097/JGP.0b013e3181953a02

Smith, E. E., Geva, A., Jonides, J., Miller, A., Reuter-Lorenz, R., \& Koeppe, R. A. (2001). The neural basis of task-switching in working memory. Proceedings of the National Academy of Sciences, USA, 98, 2095-2100. doi:10.1073/pnas.98.4.2095

Stern, Y., Habeck, C., Moeller, J., Scarmeas, N., Anderson, K. E., Hilton, ... van Heertum, R. (2005). Brain networks associated with cognitive reserve in healthy young and old adults. Cerebral Cortex, 15, 394-402. doi:10.1093/cercor/bhh142

Tombaugh, T. N., Kozak, J., \& Rees, L. (1999). Normative data stratified by age and education for two measures of verbal fluency: FAS and animal naming. Archives of Clinical Neuropsychology, 14, 167-177.

Tun, P. A., \& Lachman, M. E. (2005). The Brief Test of Adult Cognition by Telephone (BTACT). Technical report, Brandeis University.

Tun, P. A., \& Lachman, M. (2006). Telephone assessment of cognitive function in adulthood: The Brief Test of Adult Cognition by Telephone. Age and Ageing, 35, 629-632. doi:10.1093/ageing/af1095

Tun, P. A., \& Lachman, M. (2008). Age differences in reaction time in a national telephone sample of adults: Task complexity, education, and sex matter. Developmental Psychology, 44, 1421-1429. doi:10.1037/ a0012845

Umemuro, H. (2004). Computer attitudes, cognitive abilities, and technology usage among Japanese elders. Gerontechnology, 3, 64-76. doi: 10.4017/gt.2004.03.02.002.00

U.S. Department of Housing and Urban Development. (2008, June 13). About neighborhood networks. Retrieved from http://www.hud.gov/ offices/hsg/mfh/nnw/nnwaboutnn.cfm

Verghese, J., Lipton, R. B., Katz, M. J., Hall, C. B., Derby, C. A., Kuslansky, G., . . Buschke, H. (2003). Leisure activities and the risk of dementia in the elderly. New England Journal of Medicine, 348, 2508 2516. doi:10.1056/NEJMoa022252

Verhaeghen, P., \& Salthouse, T. A. (1997). Meta-analyses of age-cognition relations in adulthood: Estimates of linear and nonlinear age effects and structural models. Psychological Bulletin, 122, 231-249. doi:10.1037/ 0033-2909.122.3.231

Wechsler, D. (1997). Wechsler Adult Intelligence Scale-Third Edition. New York, NY: Psychological Corporation.

Willis, S. L. (2006). Technology and learning in current and future generations of elders. Generations, 30, 44-48.

Wilson, R. S., Bennett, D. A., Beckett, L. A., Morris, M. C., Gilley, D. W., Bienias, J. L., ... Evans, D. (1999). Cognitive activity in older persons from a geographically defined population. Journals of Gerontology, Series B: Psychological Sciences and Social Sciences, 54, 155-160.

Wilson, R. S., Mendes de Leon, C. F., Barnes, L. L., Schneider, J. A., Bienias, J. L., Evans, D., \& Bennett, D. A. (2002). Participation in cognitively stimulating activities and risk of incident Alzheimer Disease. Journal of the American Medical Association, 287, 742-748. doi: 10.1001/jama.287.6.742

Received May 19, 2009

Revision received March 9, 2010

Accepted March 18, 2010 